- Case Research.

\title{
One patient with metastastic colorectal cancer successfully treated by combination of targeted agents after failure of chemotherpay
}

Liang-Ping $\mathrm{Xia}^{1,2}$, Pei-Hong $\mathrm{Wu}^{1,3}$, Jian-Chuan $\mathrm{Xia}^{1,4}$, Bei Zhang ${ }^{1,2}$, Zhong-Zhen Guan ${ }^{1,5}$, De-Sen Wan ${ }^{1,6}$, Gui-Fang Guo ${ }^{1,2}$, Yi-Xin Zeng ${ }^{1,7}$

\begin{abstract}
${ }^{1}$ State Key Laboratory of Oncology in South China, Guangzhou, Guangdong 510060, P. R. China; ${ }^{2}$ VIP Region, Sun Yat-sen Universty Cancer Center, Guangzhou, Guangdong 510060, P. R. China; ${ }^{3}$ Department of Medical Imaging and Interventional Radiology, Sun Yat-sen Universty Cancer Center, Guangzhou, Guangdong 510060, P. R. China; ${ }^{4}$ Biotherapy Cancer, Sun Yat-sen Universty Cancer Center, Guangzhou, Guangdong 510060, P. R. China; ${ }^{5}$ Department of Medical Oncology, Sun Yat-sen Universty Cancer Center, Guangzhou, Guangdong 510060, P. R. China; ${ }^{6}$ Department of Colorectal Cancer, Sun Yat-sen Universty Cancer Center, Guangzhou, Guangdong 510060, P. R. China; ${ }^{7}$ Sun Yat-sen Universty Cancer Center, Guangzhou, Guangdong 510060, P. R. China
\end{abstract}

[Abstract】 Either cetuximab or bevacizumab can improve the survival of patients with metastastic colorectal cancer (mCRC) if administered combided with cytotoxic agents. However, the effect of two or more target agents in combination is uncertain in these patients. Here, we reported a patient with $\mathrm{mCRC}$ successfully treated by a combination of target agents after the failure of chemotherapy. The patient received palliative resection of primary tumor followed by 9 cycles of postoperative XELOX regimen, cytokine-induced killer cell (CIK)-based biotherapy, traditional Chinese medicine, particle implantation in the lung metastatic lesions. The tumor progressed 20 months after the standard treatments. Then, the regimen cetuximab, bevacizumab and cefitinib was applied. During the treatment with targeted agents, grade IV acne-like rash and relatively severe parionychia of the toes occurred. Both of them recovered smoothly. The PET-CT reexamination at 40 days after the target treatment showed that the metabolism of mediastinal lymph nodes basically recovered to a normal level. The combination of multiple targeted agents obtained a progression-free survival (PFS) of 11 months and the patient with a good quality of life during this period.

Key words: Combination, targeted agents, metastatic colorectal cancer, progression-free survival

Epidermal growth factor receptor (EGFR) regulates tumor cell growth, and vascular growth factor receptor (VEGFR) regulates the growth of vessels that transport nutrients essential for tumor cells. Thus, these receptors are important targets for molecular research and therapeutics. Studies have shown that combining cetuximab or bevacizumab and conventional chemotherapy is a successful strategy for treating metastatic colorectal cancer

Correspondence to: Yi-Xin Zeng; Tel: +86-20-87343333;

Fax: +86-20-87343333; Email: zengyx@sysucc.org.cn

This paper was translated from Chinese into English by CJC Medical Translation and edited by Wei Liu.

Received: 2010-04-10; Accepted: 2010-07-08

Grant: Major Sci-Tech Project of "National Significant New Drug Creation" (No. 2008ZX09312-002)
(CRC). First-line regimens of cetuximab plus FOLFIRI ${ }^{[1]}$ or FOLFOX ${ }^{[2]}$, for example, significantly reduced the risk of disease progression, especially in patients with wild-type KRAS gene. Combinations of cetuximab plus second-line ${ }^{[3]}$ or third-line regimens ${ }^{[4]}$ have also been effective. Similarly, therapeutic combinations with bevaciumab, including bevacizumab plus IFL as a first-line regimen ${ }^{[5]}$, bevacizumab plus FOLFOX4 as a second-line regimen ${ }^{[6]}$, and bevacizumab plus 5-fluorouracil (5-FU) or leucovorin (LV) as a third-line regimen ${ }^{[7]}$, have been extensively shown to be effective in treating metastatic CRC. Can the combination of cetuxiamb, which blocks the EGFR pathway, and bevacizumab, which blocks the VEGFR pathway, improve outcomes? Nevertheless, this issue is still unclear. Tol et al. ${ }^{[8]}$ reported that progression-free survival (PFS) decreased from 10.7 months to 9.4 months $(P=0.01)$ with a corresponding 
decrease in quality of life when cetuximab was combined with the first-line regimen of capecitabine plus oxaliplatin and bevacizumab. Similarly, no treatment benefit was found when adding panitumumab to the first-line regimen of bevacizumab plus oxaliplatin or irinotecan ${ }^{[9]}$. Based on these results, the combination of cetuximab and bevacizumab does not appear to enhance treatment efficacy. However, as both aforementioned clinical trials had used cytotoxic drugs which may confuse the real nature of combination of cetuximab and bevacizumab. In fact, there is still no direct evidence of the effect of combining cetuximab and bevacizumab. Therefore, to provide further reference for clinical practice, we now report our experience of treating one patient with advanced colorectal cancer with a pure targeted agents' combination consisted of cetuximab, bevacizumab and gefitinib at Sun Yat-sen University Cancer Center.

\section{Case report}

\section{Diagnosis}

A middle-aged man was diagnosed to have adenocarcinoma of the sigmoid colon with multiple metastases in the liver and right lung, as well as type 2 diabetes mellitus. Pathologic diagnosis was grade II-III tubular adenocarcinoma with two metastatic lymph nodes. Immunohistochemisty showed EGFR $(++)$, VEGF $(++)$, CerbB2 $(+)$, EMA $(+)$, CK20 $(++)$, CK $(+++)$, CK8 and 18 $(+++), \mathrm{CEA}(+++), \mathrm{Nm} 23(+++), \mathrm{ER}(+), \mathrm{PR}(++), \mathrm{Ki} 67(++)$, PCNA $(+++)$, P53 $(+++), B c l-2(+)$. With CD34 and F8 staining, an average of 43 interstitial capillaries in the most intensive district was observed in each high power field. Recently, we conducted related gene detection on the tumor specimen and found that the Braf gene and the 12th and 13th exons of the KRAS gene were wild-type.

\section{Disease history}

The patient received a PET-CT examination because of weight loss, intermittent pain in the left hypogastrict region, and hemafecia that occurred for half a year. The examination revealed a mass in the sigmoid colon, enlarged and highly metabolic mesenteric and para-abdominal aortic lymph nodes, as well as multiple highly metabolic foci in the liver and right lung. The patient received palliative resection of the primary tumor followed by 9 cycles of postoperative XELOX regimen, as well as other supportive treatments The disease was under control for 20 months, and no significant abnormality was found on PET-CT (Figure 1). Grade I bone marrow suppression, grade I gastrointestinal tract reaction, grade II neurotoxicity, and grade I-II hand-foot syndrome were observed during postoperative chemotherapy.

\section{Second-line treatment with multiple molecular targeted agents}

Twenty months after diagnosis, PET-CT showed enlargement and hypermetabolism in the mediastinal lymph nodes, right pulmonary hilar lymph node, and right lung lesion; thus, recurrence was considered (Figure 2). The patient rejected conventional chemotherapy. Therefore, systemic treatment with cetuximab, bevacizumab, and cefitinib was applied, in addition to radiofrequency ablation in liver metastatic lesions, particle implantation and radiofrequency ablation in lung metastatic lesions, CIK-based biotherapy, and traditional Chinese medicine. PET-CT re-examination 40 days after treatment showed that the metabolism of mediastinal lymph nodes recovered to a normal level (Figure 3). Gefitinib was ceased because of significant skin rash (after 30 days of medication), and the combination of cetuximab plus bevacizumab was maintained. PET-CT examination was subsequently conducted every 3 months, and tumor recurrence was found in the 11th month after treatment (Figure 4). Combination treatment of other targeted agents were given, however, they didn't work well.

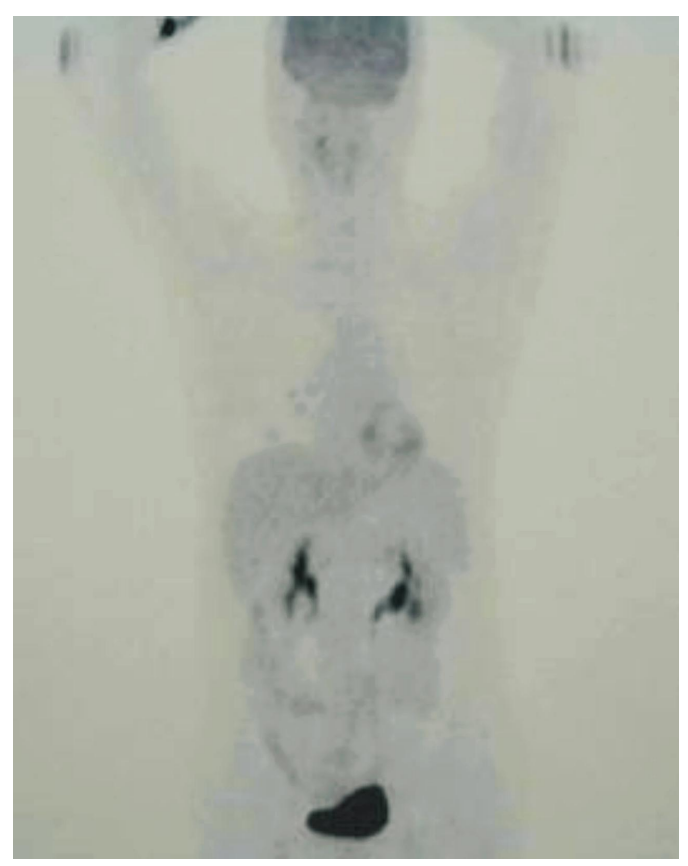

Figure 1 PET-CT reexamination on the patient with metastatic colorectal cancer at 17 months after combination treatment

The comprehensive treatment consisted of surgery, chemotherapy, $1^{125}$ particle implantation, radiofrequency ablation, and biotherapy. No sign of abnormal metabolism in the whole body is shown. 


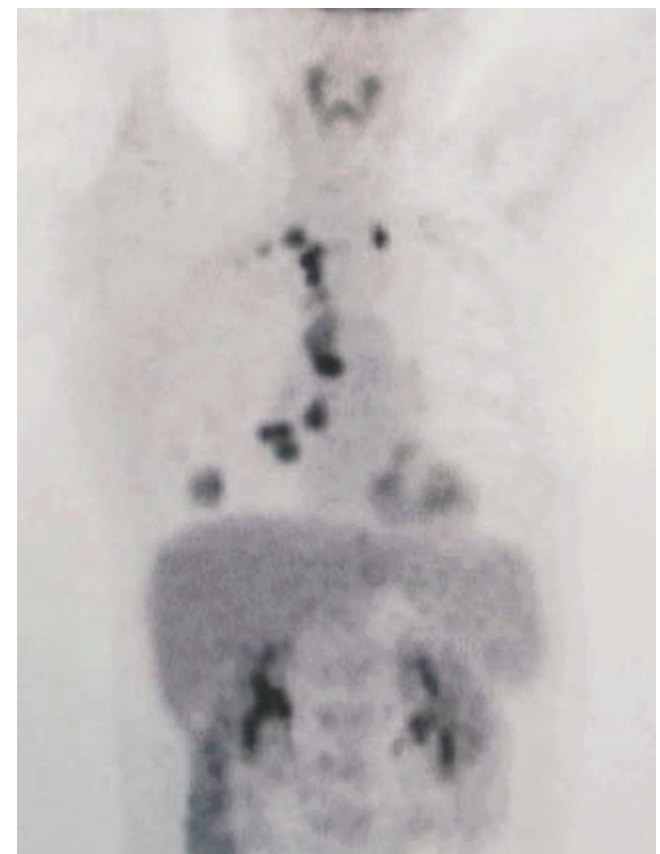

Figure 2 PET-CT reexamination on the patient with metastatic colorectal cancer at 20 months after treatment

Enlarged, highly metabolic mediastinal lymph nodes, right hilar lymph nodes, and new lesions in the liver are seen, indicating disease progression.

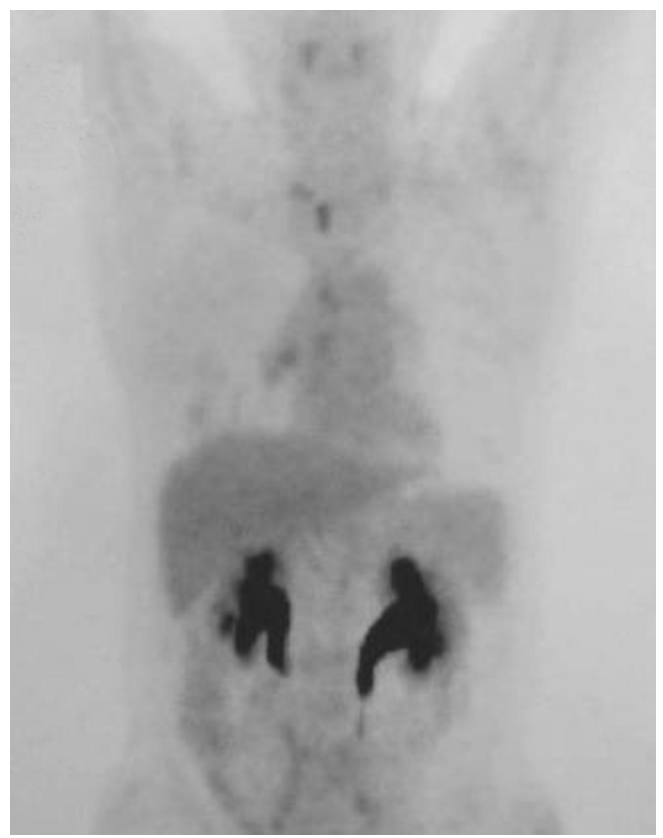

Figure 3 PET-CT reexamination on the patient with metastatic colorectal cancer at 40 days after retreatment

The metabolism of mediastinal lymph nodes nearly returned to normal after treatment with a regimen consisting of cetuximab plus bevacizumab and gefitinib, local treatment of metastatic lesions in the liver and lung, biotherapy, and traditional Chinese medicine.

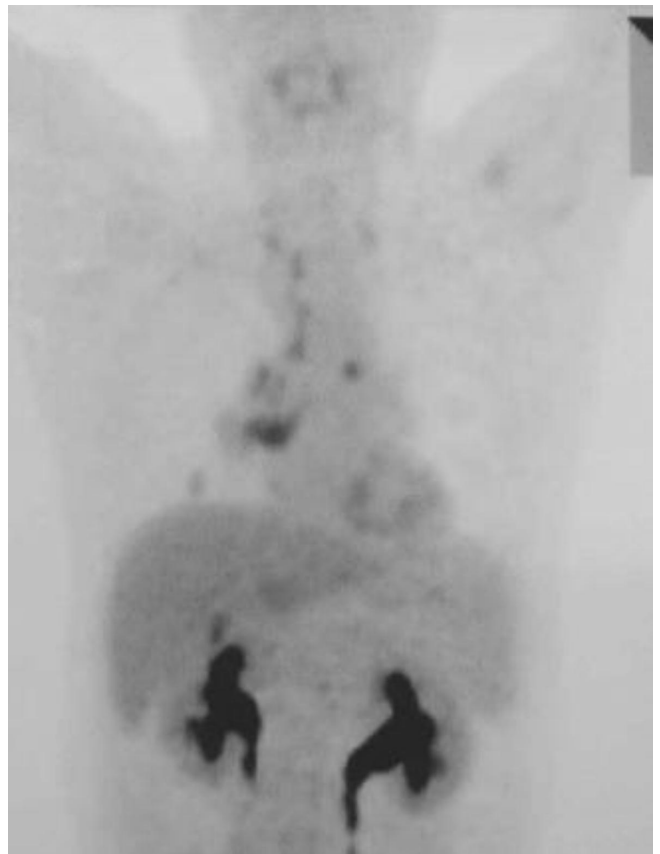

Figure 4 PET-CT reexamination on the patient with metastatic colorectal cancer at 11 months after retreatment

Recurrence in mediastinal lymph nodes is seen.

During combination therapy with multiple agents, an acne-like rash occurred. The skin rash appeared on the face at the 10th day and lasted for 2 months. It spread to upper part of the body after 1 month of medication, to the extremities after 3 months, and to the scalp (the most severe rash with fungal dermatitis, scab forming, and hemorrhage) after 5 months. After cleaning with Japanese honeysuckle flower bud and polishing with Australian rose attar, the skin rashes on the face, trunk, and extremities disappeared. Scalp rash improved after anti-inflammatory and anti-fungal treatment but reappeared after drug discontinuance and was again ameliorated when repeated medication was given. Relatively severe parionychia of the toes occurred, which recovered after dipping into $75 \%$ alcohol or iodophors.

\section{Discussion}

The surgery and first-line chemotherapy for this patient were both standard treatments; the point of most specialty lay in combined treatment with multiple molecular targeted agents after tumor recurrence.

\section{Is combined treatment with multiple molecular targeted agents reasonable?}

Combining medication is in accordance with the 
principle of pharmacology: to enhance efficacy, decrease adverse events, reduce drug-resistant cell strains, and enlarge the anticancer spectrum. EGFR function runs through the entire course of tumor growth and is, therefore, important for this process. As the tumor grows, it must depend on vessel formation to attain necessary nutrition, and VEGF plays an important role in this process. Therefore, we chose the combined regimen of bevacizumab and cetuximab for the patient. EGFR consists of intracellular, transmembrane, and extracellular domains. Hence, we chose gefitinib, which acts on the intracellular domain, and cetuximab, which acts on the extracellular domain, to simultaneously inhibit the EGFR signaling pathway to the greatest extent. Guarino et al. ${ }^{[10]}$ conducted a phase I clinical trial, during which 22 patients with solid tumors were treated with the combination of cetuximab and erlotinib. During this trial, Guarino and colleagues found that the adverse events of the combined therapy were similar to the effects of each agent when used alone. In this trial, the incidences of grade $3 / 4$ skin rash, diarrhea, and hypomagnesemia were $9 \%, 5 \%$, and $12 \%$, respectively, indicating that the combined modality is safe. Furthermore, 7 of the 18 evaluable patients achieved SD, which lasted for 16.6 weeks.

\section{Is combined treatment with multiple molecular targeted agents effective?}

Combining cetuximab or bevacizumab with conventional chemotherapy is effective in treating metastatic CRC ${ }^{[1-7]}$. However, combining EGFR monoclonal antibody and VEGF monoclonal antibody with conventional chemotherapy, including cetuximab and bevacizumab with conventional chemotherapy ${ }^{[8]}$ or bevacizumab and panitumumab with oxaliplatin/irinotecan-based chemotherapy ${ }^{[9]}$, does not enhance treatment efficacy. First, from the clinical perspective, combining bevacizumab and cetuximab with a chemotherapy regimen (combination of three agents) blocks multiple targets simultaneously, which, in the short term, increases the number of adverse events and deaths compared to combining bevacizumab with a chemotherapy regimen (combination of two agents). The 60-day mortality rate in CAIRO2 research ${ }^{[8]}$, for example, was $2.7 \%$ vs. $1.9 \%$, which influenced the PFS. The adverse events induced by the combination of three agents also increase the proportion of delay in medication. For example, the proportion of delay in PACCE research ${ }^{[9]}$ was $17 \%$ vs. $6 \%$, resulting in lowering of dose intensity. Moreover, the three agent combination also decreases median treatment cycles. In CAIRO2 research ${ }^{[8]}$, the median treatment course was 9 vs. 10, shortening the treatment duration (6 months vs. 7 months). Second, from the molecular biology perspective, molecular changes induced by single agents impact the treatment efficacy of combined therapies. One example is the differential effect of therapeutic agents on heat-inducible factor- $1 \propto(\mathrm{HIF}-1 \propto)$, an important transcription factor that regulates VEGF expression. When tumor cells are exposed to hypoxic conditions, HIF-1 $\alpha$ is induced, which then promotes tumor angiogenesis. Recently, Wang et al. ${ }^{[11]}$ found that chemotherapy down-regulated the expression of VEGF by inhibiting HIF-1 $\propto$, thereby acting synergistically with bevacizumab. Because HIF-1 $\alpha$ is a component of both the EGFR and VEGF signaling pathways, the inhibition of HIF-1 $\propto$ downstream signals by EGFR-targeting agents would also influence the efficacy of chemotherapy drugs and VEGF-targeted bevacizumab. Therefore, cetuximab weakens the effect of chemotherapy and bevacizumab ${ }^{[12]}$. Indeed, increasing the number of agents in a cytotoxic drug combination chemotherapy regimen does not always yield better results. Other studies show that the efficacy of a three-agent regimen was not better than a platinum-based two-agent regimen in the first-line treatment of lung cancer ${ }^{[13]}$, nor was a two-agent regimen better than single-agent regimen in the second-line treatment ${ }^{[14]}$. In contrast, Weihrauch et al. ${ }^{[15]}$ reported the success of combining cetuximab and bevacizumab in a single case. The patient, who, in the 7th year after colon cancer surgery, possessed huge, unresectable liver metastasis and peripheral lymph node metastasis, received surgery subsequent to neoadjuvant chemotherapy of cetuximab plus bevacizumab and XELOX regimen. With this course of treatment, the patient achieved complete response (CR) and pathologic complete response ( $\mathrm{pCR}$ ) in the liver and lymph nodes. Apparently, too many drugs have been included in PACCE and CAIRO2 research, and their interactions were quite complex. Hence, the above studies are insufficient to demonstrate that the combination of bevacizumab and cetuximab is ineffective.

Clinical experience using a combination of molecular targeted agents is limited. The BOND-2 research ${ }^{[16]}$ was the earliest attempt in this field. In that study, 83 colorectal cancer patients who experienced irinotecan treatment failure but had received neither bevacizumab nor cetuximab were randomly divided into 2 groups: cetuximab plus bevacizumab and irinotecan ( $\mathrm{CBI}$ ) group (43 patients) and cetuximab plus bevacizumab (CB) group (40 patients). For the $\mathrm{CBI}$ group, time to progression (TTP) was 7.3 months, response rate was $37 \%$, and overall survival (OS) was 14.5 months. Although the treatment outcome of the $\mathrm{CBI}$ group was better than the $C B$ group, the latter also achieved a therapeutic effect: TTP was 4.9 months, response rate was $20 \%$, and OS was 11.4 months. In addition, the adverse events in the $C B$ group were similar to those experienced when using the two drugs as single agents, indicating that the combination of cetuximab plus bevacizumab was feasible ${ }^{[16]}$. Halama et al. ${ }^{[17]}$ used bevacizumab plus 
cetuximab and irinetecan to treat 5 patients, all of whom had received cetuximab-based chemotherapy regimen many times and 2 of whom had received bevacizumab, and found the treatment was effective in 4 patients. Moosmann et al. ${ }^{[18]}$ reported the case of a single patient who, after experiencing failure of first-line chemotherapy of XELOX regimen, developed widespread liver metastases and subsequent chronic cholestasis and hyperbilirubinemia. As this patient could not receive standard irinotecan second-line treatment, Moosmann and colleagues treated the patient with a cetuximab plus bevacizumab regimen and observed quick improvement of the ECOG performance status (PS) and laboratory index, in addition to as partial response (PR) after 8 weeks ${ }^{[18]}$. Due to the recovery of the bilirubin level, the patient's course of treatment was adjusted back to the standard chemotherapy regimen of FOLFIRI plus bevacizumab ${ }^{[18]}$.

Besides intestinal cancers, research on treating other tumor types with combined multiple targeted agents are underway. A phase I/II clinical trial on treating breast cancer with a combination of trastuzumab and gefitinib as a first-line treatment showed that TTP was 3 months in all patients and 5.3 months in previously treated patients, and that OS was 27 months. These results suggest that this drug combination is safe and effective ${ }^{[19]}$. Lin et al. ${ }^{[20]}$ have also conducted a phase I clinical trial whose proposal was similar to ours. In their trial, 40 patients with advanced solid tumors were treated with the standard dosage of cetuximab combined with erlotinib, and bevacizumab was added after the maximum tolerated dose (MTD) of erlotinib was reached. They found that erlotinib $(50 \mathrm{mg} / \mathrm{d})$ plus the standard dosage of cetuximab and bevacizumab (10 mg/ $(\mathrm{kg} \cdot 2 \mathrm{w})$ ) was relatively safe. In their study, 1 patient with renal cell carcinoma achived PR and 5 patients had SD that lasted 6-11 months. The effectiveness of combining multiple targeted agents has also confirmed by experimental research. Larbouret et al. ${ }^{[21]}$, who used cetuximab combined with trastuzumab as first- and second-line regimens to treat pancreatic cancer xenografts in nude mice, found that the efficacy of combined treatment was better than gemcitabine alone and that this effect was independent of the status of KRAS gene. In HNSCC cell line, trastuzumab combined with gefitinib also enhanced treatment efficacy ${ }^{[22]}$. In animal models of thyroid cancer, cetuximab and bevacizumab used alone or in combination inhibited tumor growth and vascularization, and the efficacy of combined treatment was better than doxorubicin ${ }^{[23]}$.

\section{Challenges and prospection}

The first challenge for combination therapy is determining when and how to appropriately evaluate the treatment efficacy. Cytotoxic drugs and molecular targeted agents each have unique effects and mechanisms of killing tumor cells. Indeed, patients treated with molecular targeted agents typically experience a higher rate of SD ${ }^{[24]}$ The currently used RECIST evaluation criteria and the requirement of evaluating treatment outcome after 2 or 3 cycles of chemotherapy are not necessarily suitable for molecular targeted agent therapy. Piessevaux et al. ${ }^{[25]}$ analyzed the relationship of TTP, OS, and tumor reaction at the 6 th week in 289 patients with metastatic CRC who had received cetuximab therapy. They found that TTP was 6.1 months in patients with tumor shrinkage at the 6th week and only 1.5 months in those without shrinkage, while the OS was 13.7 months and 6.9 months, respectively. Multivariate analyses showed that tumor shrinkage within 6 weeks was a good long-term efficacy prediction index, which was even better than skin rash ${ }^{[25]}$. Presently, PET-CT scan is gradually being used to evaluate the efficacy of molecular targeted agents in lymphoma and breast cancer ${ }^{[26]}$. According to PET-CT at the 40th day after treatment, our patient experienced gPR (good partial response) to cetuximab plus bevacizumab.

The second challenge of this study was that intestinal cancer has many possible targets or affected signaling pathways. Our patient, in particular, had simultaneously detectable levels of EGFR, VEGF, CerbB2, EMA, CK20, CK, CK8, CK18, CEA, Nm23, ER, PR, Ki67, PCNA, P53, and $\mathrm{Bcl}-2$, making it difficult to surmise which would be effective targets. Adding further complexity was the knowledge that the role of a single pathway varies in different tumors. For example, the EGFR pathway plays a key role in non-small cell lung cancer (NSCLC) and is relatively exclusive ${ }^{[27,28]}$; therefore, tyrosine kinase inhibitor (TKI) alone could potentially yield a satisfactory treatment outcome. On the other hand, although the EGFR pathway is quite important in intestinal cancer, the effect of cetuximab alone in first-line treatment is rather limited ${ }^{[29]}$. Instead, the mutation of KRAS gene determines the efficacy of cetuximab on intestinal cancer, though KRAS status has no influence on the efficacy of cetuximab in NSCLC ${ }^{[30]}$.

\section{Conclusion}

We report here the novel regimen consisted of cetuximab plus bevacizumab and gefitinib achieved a PFS of 11 months after the failure of first-line standard chemotherapy for a patient with $\mathrm{mCRC}$. Our pure targeted agents' combination may challenge clinical trails of CAIRO2 and PACCE since both of them denied the combination of cetuximab and bevacizumab under the confusion of cytotoxic agents. 


\section{References}

[1] Van Cutsem E, Köhne $\mathrm{CH}$, Hitre E, et al. Cetuximab and chemotherapy as initial treatment for metastatic colorectal cancer [J]. N Engl J Med, 2009,360(14):1408-1417.

[2] Bokemeyer C, Bondarenko I, Makhson A, et al. Fluorouracil, leucovorin, and oxaliplatin with and without cetuximab in the firstline treatment of metastatic colorectal cancer [J]. J Clin Oncol, 2009, 27(5):663-671.

[3] Sobrero AF, Maurel J, Fehrenbacher L, et al. EPIC: phase III trial of cetuximab plus irinotecan after fluoropyrimidine and oxaliplatin failure in patients with metastatic colorectal cancer [J]. J Clin Oncol, 2008, 26(14):2311-2319.

[4] Cunningham D, Humblet $Y$, Siena S, et al. Cetuximab monotherapy and cetuximab plus irinotecan in irinotecan-refractory metastatic colorectal cancer [J]. N Engl J Med, 2004, 351(4):337-345.

[5] Hurwitz H, Fehrenbacher L, Novotny W, et al. Bevacizumab plus irinotecan, fluorouracil, and leucovorin for metastatic colorectal cancer [J]. N Engl J Med, 2004, 350: 2335-2342.

[6] Giantonio BJ, Catalano PJ, Meropol NJ, et al. Bevacizumab in combination with oxaliplatin, fluorouracil, and leucovorin (FOLFOX4) for previously treated metastatic colorectal cancer: results from the Eastern Cooperative Oncology Group Study E3200 [J]. J Clin Oncol, 2007, 25(12):1539-1544.

[7] Chen HX, Mooney M, Boron M, et al. Phase II multicenter trial of bevacizumab plus fluorouracil and leucovorin in patients with advanced refractory colorectal cancer: an $\mathrm{NCl}$ Treatment Referral Center Trial TRC-0301 [J]. J Clin Oncol, 2006,24(21):3354-3360.

[8] Tol J, Koopman M, Cats A, et al. Chemotherapy, bevacizumab, and cetuximab in metastatic colorectal cancer [J]. N Engl J Med, 2009, 360(6):563-572

[9] Hecht JR, Mitchell E, Chidiac T, et al. A randomized phase IIIB trial of chemotherapy, bevacizumab, and panitumumab compared with chemotherapy and bevacizumab alone for metastatic colorectal cancer [J]. J Clin Oncol, 2009, 27(5):672-680.

[10] Guarino MJ, Schneider CJ, Hosford MA, et al. Dual inhibition of the epidermal growth factor receptor pathway with cetuximab and erlotinib: a phase I study in patients with advanced solid malignancies [J]. Oncologist, 2009, 14(2):119-124.

[11] Wang $Y, X u ~ J M, X u ~ Q Z$, et al. Combination with SN-38 on human colon cancer LoVo cells [J]. Chin J Oncol, 2009,31(10):746-851. [in Chinese]

[12] Xu JM. Several issues faced by molecular targeted therapy in colorectal cancer [J]. Chin J Oncol, 2009,31 (5):321-323. [in Chinese]

[13] Mountzios G, Soria JC. Advanced non-small-cell lung cancer: "triplets" better than "doublets"? [J]. Nat Clin Pract Oncol, 2006, 3(9):476-477.

[14] Zhang YF, Chen ZW, Lu S. Pemetrexed monotherapy versus pemetrexed plus platinum combination as second-line treatment for advanced non-small cell lung cancer [J]. J Chin Med, 2009, 122 (20):2472-2476

[15] Weihrauch MR, Stippel D, Fries JW, et al. Complete remission in a colon cancer patient with a large, irresectable liver metastasis after
XELOX/cetuximab/bevacizumab treatment [J]. Onkologie, 2008, 31 (8-9):464-467.

[16] Saltz LB, Lenz HJ, Kindler HL, et al. Randomized phase II trial of cetuximab, bevacizumab, and irinotecan compared with cetuximab and bevacizumab alone in irinotecan-refractory colorectal cancer: the BOND-2 study [J]. J Clin Oncol, 2007, 25(29):4557-4561.

[17] Halama N, Herrmann C, Jaeger D, et al. Treatment with cetuximab, bevacizumab and irinotecan in heavily pretreated patients with metastasized colorectal cancer [J]. Anticancer Res, 2008, 28(6B):4111-4115

[18] Moosmann N, Laessig D, Michaely HJ, et al. Effective second-line treatment with cetuximab and bevacizumab in a patient with hepatic metastases of colorectal cancer and hyperbilirubinemia [J]. Onkologie, 2007, 30(10):509-512.,

[19] Arteaga CL, O'Neill A, Moulder SL, et al. A phase I-II study of combined blockade of the ErbB receptor network with trastuzumab and gefitinib in patients with HER2 (ErbB2)-overexpressing metastatic breast cancer [J]. Clin Cancer Res, 2008, 14(19):62776283.

[20] Lin CC, Calvo E, Papadopoulos KP, et al. Phase I study of cetuximab, erlotinib, and bevacizumab in patients with advanced solid tumors [J]. Cancer Chemother Pharmacol, 2009, 63(6):10651071.

[21] Larbouret C, Robert B, Bascoul-Mollevi C, et al. Combined cetuximab and trastuzumab are superior to gemcitabine in the treatment of human pancreatic carcinoma xenografts [J]. Ann Oncol, 2010, 21(1):98-103.

[22] Kondo N, Ishiguro $\mathrm{Y}$, Kimura $\mathrm{M}$, et al. Antitumor effect of gefitinib on head and neck squamous cell carcinoma enhanced by trastuzumab [J]. Oncol Rep, 2008, 20(2):373-378.

[23] Prichard CN, Kim S, Yazici YD, et al. Concurrent cetuximab and bevacizumab therapy in a murine orthotopic model of anaplastic thyroid carcinoma [J]. Laryngoscope, 2007, 117(4):674-679.

[24] Llovet JM, Ricci S, Mazzaferro V, et al. Sorafenib in advanced hepatocellular carcinoma [J]. N Engl J Med, 2008, 359 (4):378390.

[25] Piessevaux H, Buyse M, De Roock W, et al. Radiological tumor size decrease at week 6 is a potent predictor of outcome in chemorefractory metastatic colorectal cancer treated with cetuximab (BOND trial) [J]. Ann Oncol, 2009, 20(8): 1375-1382.

[26] Juweid ME, Cheson BD. Positron-emission tomography and assessment of cancer therapy [J]. N Engl J Med, 2006, 354 (5): 496-507.

[27] Chitale D, Gong Y, Taylor BS, et al. An integrated genomic analysis of lung cancer reveals loss of DUSP4 in EGFR-mutant tumors [J]. Oncogene, 2009, 28(31):2773-2783.

[28] Ding L, Getz G, Wheeler DA, et al. Somatic mutations affect key pathways in lung adenocarcinoma [J]. Nature, 2008, 455(7216): 1069-1075.

[29] Pessino A, Artale S, Sciallero S, et al. First-line single-agent cetuximab in patients with advanced colorectal cancer [J]. Ann Oncol, 2008, 19(4):711-716.

[30] Mack PC, Holland WS, Redman M, et al. KRAS mutation analysis in cetuximab-treated advanced stage non-small cell lung cancer (NSCLC): SWOG experience with S0342 and S0536 [J]. ASCO Meeting Abstracts, May 20 2009: 8022. 